\title{
REPRESENTATIONS OF FINITE GROUPS AND CUNTZ-KRIEGER ALGEBRAS
}

\author{
M.H. Mann, IaIn RaEburn and C.E. Sutherland
}

\begin{abstract}
We investigate the structure of the $C^{*}$-algebras $\mathcal{O}_{\rho}$ constructed by Doplicher and Roberts from the intertwining operators between the tensor powers of a representation $\rho$ of a compact group. We show that each Doplicher-Roberts algebra is isomorphic to a corner in the Cuntz-Krieger algebra $\mathcal{O}_{A}$ of a $\{0,1\}$-matrix $A=A_{\rho}$ associated to $\rho$. When the group is finite, we can then use Cuntz's calculation of the $K$-theory of $\mathcal{O}_{A}$ to compute $K_{*}\left(\mathcal{O}_{\rho}\right)$.
\end{abstract}

Doplicher and Roberts have recently developed a duality theory for compact subgroups of $S U(n, \mathrm{C})$ in which the dual object consists of a simple $C^{*}$-algebra $\mathcal{O}_{G}$ and an endomorphism of $\mathcal{O}_{G}[3,4]$. The construction of $\mathcal{O}_{G}$ is based on the concrete representation $\rho$ of $G$ in $S U(n, C)$ rather than the abstract group $G$, so we prefer to call it $\mathcal{O}_{\rho}$; our work originated in an attempt to find out how the structure of $\mathcal{O}_{\rho}$ depends on the choice of representation. To this end we have computed the $K$-theory of $\mathcal{O}_{\rho}$ for finite $G$, by embedding it as a corner in a Cuntz-Krieger algebra $\mathcal{O}_{A}$, and using Cuntz's calculation of $K_{*}\left(\mathcal{O}_{A}\right)$ [1]. One conclusion is that different representations of the same finite group can give algebras which have quite different $K$-theory, and hence are not even stably isomorphic or Morita equivalent.

The algebra $\mathcal{O}_{\rho}$ is constructed from the spaces of intertwining operators between the different tensor powers $\rho^{n}$ of $\rho$, and its structure is determined by the decompositions of $\rho^{n}$ into irreducibles, and hence by the decompositions of $\pi \otimes \rho$ for $\pi \in \hat{G}$. The combinatorics of the situation can be summed up in a bipartite graph with $\widehat{G}$ as vertices, and our main observation is that these combinatorics are similar to those involved in Cuntz and Krieger's construction of a $C^{*}$-algebra $\mathcal{O}_{A}$ from a $\{0,1\}$-matrix $A$. When $G$ is compact, $A$ is infinite, and there are technical problems in transferring this combinatorial similarity to the $C^{*}$-algebra level; indeed, we need to appeal to both [2] and [3] to do it. For finite groups, we can prove directly that $\mathcal{O}_{\rho}$ is a corner in $\mathcal{O}_{A}$, and the simplicity of $\mathcal{O}_{\rho}$ therefore follows from [2] alone. We shall go as far as we can in full generality, since we are optimistic that one can extend the results of [1] to cover infinite $A$, and use them to compute $K_{*}\left(\mathcal{O}_{\rho}\right)$ for compact $G$ along similar lines.

Received 6th September, 1991

Copyright Clearance Centre, Inc. Serial-fee code: 0004-9729/92 \$A2.00+0.00. 
We begin with a discussion of the two Doplicher-Roberts algebras ${ }^{0} \mathcal{O}_{\rho}, \mathcal{O}_{\rho}$ associated to a finite-dimensional representation $\rho:{ }^{0} \mathcal{O}_{\rho}$ is a ${ }^{*}$-algebra, and $\mathcal{O}_{\rho}$ its $C{ }^{*}$. enveloping algebra. In Section 2 , we associate a $\{0,1\}$-matrix $A_{\rho}$ to $\rho$, and show how ${ }^{0} \mathcal{O}_{\rho}$ can be canonically mapped into the Cuntz-Krieger algebra $\mathcal{O}_{A_{\rho}}$; in Section 3 , we prove that, when $G$ is finite, this mapping induces an isomorphism of $\mathcal{O}_{\rho}$ onto a corner $P \mathcal{O}_{A_{\rho}} P$ in $\mathcal{O}_{A_{\rho}}$. Since $\mathcal{O}_{A_{\rho}}$ is known to be simple [2], this implies that $\mathcal{O}_{\rho}$ is Morita equivalent to $\mathcal{O}_{A_{\rho}}$, and in particular has the same $K$-theory. In our final section, we compute $K_{*}\left(\mathcal{O}_{\rho}\right)$ for a few examples of finite groups, using methods which should work whenever we have a character table for $G$.

One could also hope to investigate the structure of Doplicher-Roberts algebras by realising them as the $C^{*}$-algebras of locally compact groupoids whose unit spaces are path spaces associated to the infinite diagram of Section 1 , and exploiting general properties of groupoid $C^{*}$-algebras, as done for $A F$-algebras in [7]. At present, though, it is not clear whether the appropriate groupoids for the Cuntz-Krieger algebras of infinite $\{0,1\}$-matrices are locally compact, and hence the present approach may be more easily adapted to compact groups. In [6], we gave a brief discussion of the groupoid approach, and the problems involved in it.

We stress that many of the ideas and results in this paper are either well-known or implicit in the work of Doplicher-Roberts and Cuntz-Krieger. For example, our comments in Section 4 on computing $K_{*}\left(\mathcal{O}_{A}\right)$ are surely known to all experts. However, we do hope a detailed presentation of this circle of ideas in a technically-straightforward special case will be informative and useful.

This research was supported by the Australian Research Council.

\section{Doplicher-Roberts algebras}

Let $\rho$ be a finite-dimensional representation of a locally compact group, and for $n \in$ $\mathbf{N}$, let $\rho^{n}$ be the $n$-fold tensor power of $\rho$, acting in $H_{\rho} \otimes \cdots \otimes H_{\rho}=H_{\rho}^{n}$. For each pair $m, n \in \mathrm{N}$, we denote by $\left(\rho^{m}, \rho^{n}\right)$ the space of intertwining operators $T: H_{\rho}^{n} \rightarrow H_{\rho}^{m}$; we have chosen this notation so that the composition $S \circ T$ of $S \in\left(\rho^{m}, \rho^{n}\right), T \in\left(\rho^{n}, \rho^{p}\right)$ lies in $\left(\rho^{m}, \rho^{p}\right)$. There is a natural embedding $T \rightarrow T \otimes 1$ of $\left(\rho^{m}, \rho^{n}\right)$ in $\left(\rho^{m+1}, \rho^{n+1}\right)$, and we denote the direct $\operatorname{limit} \underset{\longrightarrow}{\lim }\left(\rho^{p}, \rho^{p+k}\right)$ by ${ }^{0} \mathcal{O}_{\rho}^{k}$. The direct sum ${ }^{0} \mathcal{O}_{\rho}=\bigoplus_{k \in \mathbf{Z}}{ }^{0} \mathcal{O}_{\rho}^{k}$ is a *-algebra in which the product of $S \in\left(\rho^{m}, \rho^{n}\right)$ and $T \in\left(\rho^{p}, \rho^{q}\right)$ is

$$
\begin{cases}\left(S \otimes 1_{p-n}\right) \circ T \in\left(\rho^{m+(p-n)}, \rho^{q}\right) & \text { if } p \geqslant n \\ S \circ\left(T \otimes 1_{n-p}\right) \in\left(\rho^{m}, \rho^{q+(n-p)}\right) & \text { if } p>n,\end{cases}
$$

and the adjoint of $S \in\left(\rho^{m}, \rho^{n}\right)$ is $S^{*} \in\left(\rho^{n}, \rho^{m}\right)$.

We shall refer to either ${ }^{0} \mathcal{O}_{\rho}$ or its $C^{*}$-enveloping algebra $\mathcal{O}_{\rho}$ as a Doplicher-Roberts algebra; of course, it is not immediately obvious that ${ }^{0} \mathcal{O}_{\rho}$ has a $C^{*}$-enveloping algebra, 
since a priori

$$
\|T\|=\sup \left\{\|\pi(T)\|: \pi \text { is a }{ }^{*} \text {-representation of }{ }^{0} \mathcal{O}_{\rho}\right\}
$$

could be infinite. To settle this, we shall describe a natural basis for each $\left(\rho^{m}, \rho^{n}\right)$, which is parametrised by paths in an infinite graph associated to $\rho$, and which will be important in our later constructions.

We first let $R$ be the set of (equivalence classes of) irreducible summands of the tensor powers $\rho^{n}$, (adding in the trivial representation $\iota$, if necessary), and to each element of $R$ we associate a specific representation $\pi: G \rightarrow U\left(H_{\pi}\right)$. We define a bipartite graph with $R$ as the set of vertices, and the number of edges joining $\pi_{1}$ at the top level to $\pi_{2}$ at the lower level equal to the multiplicity of $\pi_{2}$ in $\pi_{1} \otimes \rho$. Thus, for example, if $\pi_{2}$ occurs with multiplicity 2 in $\pi_{1} \otimes \rho$, and multiplicity 1 in $\pi_{3} \otimes \rho$, the graph contains

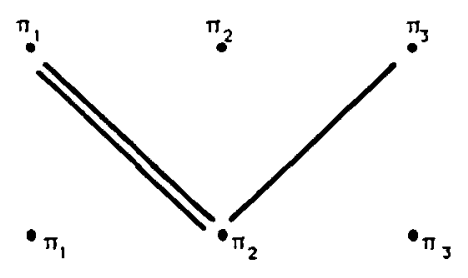

If $x$ is an edge from $\pi_{1}$ above to $\pi_{2}$ below, we write $s(x)=\pi_{1}$ and $r(x)=\pi_{2}$, and we let $E$ denote the set of all edges. We now assign to each edge $x$ an isometric intertwiner $T_{x}: H_{r(x)} \rightarrow H_{\wedge(x)} \otimes H_{\rho}$, in such a way that, for each $\pi$,

$$
H_{\pi} \otimes H_{\rho}=\bigoplus_{\{x: \rho(x)=\pi\}} T_{x} T_{x}^{*}\left(H_{\pi} \otimes H_{\rho}\right)
$$

-in other words, such that the edges out of $\pi$ give a specific decomposition of $H_{\pi} \otimes H_{\rho}$ into irreducibles. Next we consider the infinite graph obtained by sticking infinitely many copies of the bipartite graph below the original. We note that a sequence $x_{1}, x_{2}, \ldots, x_{n}$ of edges in the original graph combines to form a vertical path in the infinite graph if and only if $r\left(x_{j}\right)=s\left(x_{j+1}\right)$ for all $j$. Each path $x=\left\{x_{1}, x_{2}, \ldots x_{n}\right\}$ represents an intertwiner

$$
T_{x}=\left(T_{x_{1}} \otimes 1_{n-1}\right) \circ\left(T_{x_{2}} \otimes 1_{n-2}\right) \circ \cdots \circ T_{x_{n}}: H_{r\left(x_{n}\right)} \rightarrow H_{\rho}^{n},
$$

where $1_{r}$ denotes the identity operator on $H_{\rho}^{r}$, and the paths $x$ with $s\left(x_{1}\right)=\iota$ provide an explicit decomposition of $H_{\rho}^{n}$ into irreducibles:

$$
H_{\rho}^{n}=\bigoplus_{\left\{\text {paths } x \text { with } s\left(x_{1}\right)=\iota\right\}} T_{x} T_{x}^{*}\left(H_{\rho}^{n}\right) .
$$




\section{Proposition 1.1. The family}

$$
\left\{T_{x} T_{y}^{*}:|x|=m|y|=n, s\left(x_{1}\right)=s\left(y_{1}\right)=\iota, r\left(x_{m}\right)=r\left(y_{n}\right)\right\}
$$

is a basis for $\left(\rho^{m}, \rho^{n}\right)$, and each basis element $T_{x} T_{y}^{*}$ is a partial isometry.

Proof: Each pair of paths $x, y$ with $|x|=m,|y|=n$ and $s\left(x_{1}\right)=\iota=s\left(y_{1}\right)$ determines a pair of irreducible summands $T_{x}\left(H_{r\left(x_{m}\right)}\right), T_{y}\left(H_{r\left(y_{n}\right)}\right)$ of $H_{\rho}^{m}, H_{\rho}^{n}$; the space of intertwiners of these representations is 0 unless $r\left(x_{m}\right)=r\left(y_{n}\right)$, and then is the 1-dimensional space spanned by $T_{x} T_{y}^{*}$. Hence every intertwiner in $\left(\rho^{m}, \rho^{n}\right)$ can be uniquely expressed as a linear combination of the $T_{x} T_{y}^{*}$, as claimed. Because each $T_{y}$ is isometric, $T_{y}^{*}$ is a partial isometry with range space $H_{r\left(y_{n}\right)}$, and, whenever $r\left(x_{m}\right)=r\left(y_{n}\right), T_{x} T_{y}^{*}$ is also a partial isometry.

Corollary 1.2. For every $T \in{ }^{0} \mathcal{O}_{\rho}$,

$$
\|T\|=\sup \left\{\|\pi(T)\|: \pi \text { is a }{ }^{*} \text {-representation of }{ }^{0} \mathcal{O}_{\rho}\right\}
$$

is finite.

Proof: As every element of ${ }^{0} \mathcal{O}_{\rho}$ is a finite sum of elements of ${ }^{0} \mathcal{O}_{\rho}^{k}$, and each of these is a direct limit, we may as well suppose that $T \in\left(\rho^{m}, \rho^{n}\right)$, and hence that $T$ can be uniquely written as a linear combination $\sum \lambda_{x, y} T_{x} T_{y}^{*}$. Now an operator $S \in\left(\rho^{m}, \rho^{n}\right)$ is a partial isometry if and only if $S=S S^{*} S$ as operators on $H_{\rho}^{n}$, and hence, by definition of the *-algebra structure on ${ }^{0} \mathcal{O}_{\rho}$, if and only if $S=S S^{*} S$ in ${ }^{0} \mathcal{O}_{\rho}$. Thus $\pi\left(T_{x} T_{y}^{*}\right)$ is a partial isometry for every representation $\pi$ of ${ }^{0} \mathcal{O}_{\rho}$, and

$$
\|T\| \leqslant \sum\left|\lambda_{x, y}\right|\left\|\pi\left(T_{x} T_{y}^{*}\right)\right\| \leqslant \sum\left|\lambda_{x, y}\right|
$$

which gives the Corollary.

REMARK. Although we have not insisted that the group $G$ be compact, as Doplicher and Roberts do, the extra generality is spurious: if $\rho$ is finite-dimensional, the intertwining spaces for the identity representation $\iota_{K}$ of the compact group $K=\overline{\rho(\bar{G})} \subset U\left(H_{\rho}\right)$ are exactly the same as those of $\rho$, and hence $\mathcal{O}_{\iota K}=\mathcal{O}_{\rho}$. However, there are non-compact groups with lots of finite-dimensional representations - for example, $S L(2, \mathbf{Z}) \cong \mathbf{Z}_{2} * \mathbf{Z}_{3}$, and the integer Heisenberg group - and there could possibly be interesting interplay between the combinatorics of the representation, the algebra $\mathcal{O}_{\rho}$, and the underlying non-compact group.

\section{Representing a Doplicher-Roberts algebra in a Cuntz-Krieger algebra}

Again let $\rho$ be a finite-dimensional representation of a locally compact group, and resume the notation of the previous section. Define a (possibly infinite) $\{0,1\}$-matrix 
$A_{\rho}$, indexed by the set $E$ of edges in the bipartite graph associated to $\rho$, as follows:

$$
A_{\rho}(x, y)= \begin{cases}1 & \text { if } r(x)=s(y) \\ 0 & \text { otherwise }\end{cases}
$$

THEOREM 2.1. Let $\rho$ be a finite-dimensional representation of a locally compact group, and use the notation of Section 1. Let $\left\{S_{x}: x \in E\right\}$ be a family of non-zero partial isometries satisfying

$$
S_{x}^{*} S_{x}=\sum_{y \in E} A_{p}(x, y) S_{y} S_{y}^{*}
$$

let $B$ be the *-algebra generated by $\left\{S_{x}\right\}$, and let

$$
P=\sum_{\{x \in E: \Delta(x)=\iota\}} S_{x} S_{x}^{*}
$$

Then there is a ${ }^{*}$-homomorphism of the Doplicher-Roberts algebra ${ }^{0} \mathcal{O}_{\rho}$ onto the corner $P B P$.

The idea is that paths in the infinite diagram of Section 1 have interpretations in the Cuntz-Krieger algebra $C^{*}\left(S_{x}\right)$, as well as the Doplicher-Roberts algebra ${ }^{0} \mathcal{O}_{\rho}$. A sequence $x_{1}, x_{2}, \ldots, x_{n}$ of edges in the original graph combines to form a vertical path in the infinite graph if and only if $r\left(x_{j}\right)=s\left(x_{j+1}\right)$ for all $j$, hence if and only if $A_{p}\left(x_{j}, x_{j+1}\right)=1$ for all $j$, and hence exactly when the the product $S_{x}=S_{x_{1}} S_{x_{2}} \ldots S_{z_{n}}$ is non-zero [2, p.252]. And, parallel to Lemma 1.1, every element of $B$ is a linear combination of operators $S_{x} S_{y}^{*}$ with $r\left(x_{m}\right)=r\left(y_{n}\right)$.

We now define $\phi_{m, n}:\left(\rho^{m}, \rho^{n}\right) \rightarrow B$ by $\phi_{m, n}\left(T_{x} T_{y}^{*}\right)=S_{x} S_{y}^{*}$. Notice that, since $s\left(x_{1}\right)=s\left(y_{1}\right)=\iota$, we have

$$
S_{x} S_{y}^{*}=S_{z_{1}} S_{x_{1}}^{*}\left(S_{z} S_{y}^{*}\right) S_{y_{1}} S_{y_{1}}^{*}=P\left(S_{x_{1}} S_{x_{1}}^{*}\right)\left(S_{x} S_{y}^{*}\right)\left(S_{y_{1}} S_{y_{1}}^{*}\right) P=P S_{z} S_{y}^{*} P
$$

and hence $\phi_{m, n}:\left(\rho^{m}, \rho^{n}\right) \rightarrow P B P$. We claim that the maps $\phi_{m, n}$ are compatible with the bonding maps $\left(\rho^{m}, \rho^{n}\right) \rightarrow\left(\rho^{m+1}, \rho^{n+1}\right)$, in the sense that

$$
\phi_{m+1, n+1}\left(\left(T_{z} T_{y}^{*}\right) \otimes 1\right)=\phi_{m, n}\left(T_{x} T_{y}^{*}\right) .
$$

To see this, we note that

$$
H_{r\left(x_{m}\right)} \otimes H_{\rho}=\bigoplus_{\left\{z \in E: \Delta(z)=r\left(x_{m}\right)\right\}} T_{z} T_{z}^{*}\left(H_{r\left(x_{m}\right)} \otimes H_{\rho}\right)
$$


so that

$$
\begin{aligned}
T_{x} T_{y}^{*} \otimes 1 & =\sum_{\left\{z: \Delta(z)=r\left(x_{m}\right)=r\left(y_{n}\right)\right\}}\left(T_{x} \otimes 1\right)\left(T_{z} T_{z}^{*}\right)\left(T_{y}^{*} \otimes 1\right) \\
& =\sum_{\left\{z: \Delta(z)=r\left(x_{m}\right)=r\left(y_{n}\right)\right\}}\left(T_{x z}\right)\left(T_{y z}\right)^{*} ;
\end{aligned}
$$

on the other hand,

$$
\begin{aligned}
S_{x} S_{y}^{*} & =S_{x}\left(S_{x_{m}}^{*} S_{x_{m}}\right) S_{y}^{*} \\
& =S_{x}\left(\sum_{z \in E} A\left(x_{m}, z\right) S_{z} S_{z}^{*}\right) S_{y}^{*} \\
& =\sum_{\left\{z: s(z)=r\left(x_{m}\right)\right\}} S_{x}\left(S_{z} S_{z}^{*}\right) S_{y}^{*} \\
& =\sum_{\left\{z: s(z)=r\left(x_{m}\right)\right\}}\left(S_{x z}\right)\left(S_{y z}\right)^{*}
\end{aligned}
$$

and (2.2) follows.

We can now define $\phi=\oplus \phi^{k}$, at least as a linear map, and we have to verify that $\phi$ is a *-homomorphism. Well,

$$
\phi_{m, n}\left(T_{x} T_{y}^{*}\right)^{*}=\left(S_{x} S_{y}^{*}\right)^{*}=S_{y} S_{x}^{*}=\phi_{n, m}\left(T_{y} T_{x}^{*}\right)
$$

so $\phi$ is certainly *-preserving. To check that $\phi$ is multiplicative, consider $T_{x} T_{y}^{*} \in$ $\left(\rho^{m}, \rho^{n}\right), T_{w} T_{z}^{*} \in\left(\rho^{p}, \rho^{q}\right)$, and suppose for the sake of argument that $p \geqslant n$. Then

$$
\phi\left(\left(T_{x} T_{y}^{*}\right)\left(T_{w} T_{z}^{*}\right)\right)=\phi^{(n-m)+(q-p)}\left(\left(\left(T_{x} T_{y}^{*}\right) \otimes 1_{p-n}\right) \circ\left(T_{w} T_{z}^{*}\right)\right)
$$

The product $\left(T_{y}^{*} \otimes 1_{p-n}\right) T_{w}$ is by definition the composition

$$
\left(T_{y_{n}}^{*} \otimes 1_{p-n}\right) \circ\left(T_{y_{n-1}}^{*} \otimes 1_{p-n+1}\right) \circ \cdots \circ\left(T_{y_{1}}^{*} \otimes 1_{p-1}\right) \circ\left(T_{w_{1}} \otimes 1_{p-1}\right) \circ \cdots \circ T_{w_{p}}
$$

Since

$$
T_{y_{1}}^{*} T_{w_{1}}= \begin{cases}0 & \text { unless } w_{1}=y_{1} \\ T_{y_{1}}^{*} T_{y_{1}}=1 & \text { if } w_{1}=y_{1},\end{cases}
$$

and we know $y$ is a path, $T_{y_{2}}^{*}\left(\left(T_{y_{1}}^{*} T_{y_{1}}\right) \otimes 1\right)=T_{y_{2}}^{*}$; thus we can omit the two middle terms in $\left(T_{y}^{*} \otimes 1\right) T_{w}$. By induction, we deduce that the composition is 0 unless $y_{i}=w_{i}$ for $1 \leqslant i \leqslant n$, and then equals

$$
\left(T_{w_{n+1}} \otimes 1_{p-n-1}\right) \circ \cdots \circ T_{w_{n}}=T_{w^{\prime}}
$$


say. Thus

$$
\begin{aligned}
\left(\left(T_{x} T_{y}^{*}\right) \otimes 1_{p-n}\right) T_{w} T_{z}^{*} & = \begin{cases}\left(T_{x} \otimes 1_{p-n}\right) \circ T_{w^{\prime}} \circ T_{z}^{*} & \text { if } y_{i}=w_{i} \text { for } 1 \leqslant i \leqslant n \\
0 & \text { otherwise, }\end{cases} \\
& = \begin{cases}T_{x w^{\prime}} T_{z}^{*} & \text { if } y_{i}=w_{i} \text { for } 1 \leqslant i \leqslant n \\
0 & \text { otherwise. }\end{cases}
\end{aligned}
$$

But this is precisely the rule for cancelling $S_{y}^{*} S_{w}$ :

$$
\begin{aligned}
S_{z}\left(S_{y}^{*} S_{w}\right) S_{z}^{*} & = \begin{cases}S_{x}\left(S_{y_{n}}^{*} S_{y_{n}}\right) S_{w^{\prime}} S_{z}^{*} & \text { if } y_{i}=w_{i} \text { for } 1 \leqslant i \leqslant n \\
0 & \text { otherwise }\end{cases} \\
& = \begin{cases}S_{x} S_{w^{\prime}} S_{z}^{*} & \text { if } y_{i}=w_{i} \text { for } 1 \leqslant i \leqslant n \\
0 & \text { otherwise, }\end{cases}
\end{aligned}
$$

since $r\left(x_{m}\right)=r\left(y_{n}\right)$. Hence $\phi$ is multiplicative, as claimed.

The algebra $B$ is spanned by the elements of the form $S_{x} S_{y}^{*}$, which is non-zero only if there exists $z$ with $A\left(x_{m}, z\right)=A\left(y_{n}, z\right)=1$, that is, only if $r\left(x_{m}\right)=r\left(y_{n}\right)$. Since

$$
P S_{x} S_{y}^{*} P= \begin{cases}S_{x} S_{y}^{*} & \text { if } s\left(x_{1}\right)=\iota \text { and } s\left(y_{1}\right)=\iota \\ 0 & \text { otherwise }\end{cases}
$$

it follows that

$$
\begin{aligned}
P S_{x} S_{y}^{*} P & = \begin{cases}S_{x} S_{y}^{*} & \text { if } s\left(x_{1}\right)=\iota=s\left(y_{1}\right) \text { and } r\left(x_{m}\right)=r\left(y_{n}\right) \\
0 & \text { otherwise, }\end{cases} \\
& = \begin{cases}\phi_{m, n}\left(T_{x} T_{y}^{*}\right) & \text { if } s\left(x_{1}\right)=\iota=s\left(y_{1}\right) \text { and } r\left(x_{m}\right)=r\left(y_{n}\right) \\
0 & \text { otherwise. }\end{cases}
\end{aligned}
$$

Thus the non-zero operators of the form $P S_{x} S_{y}^{*} P$ are all in the range of $\phi$, and since they span $P B P$, the homomorphism $\phi$ maps onto $P B P$.

This completes the proof of Theorem 2.1.

Corollary 2.2. There is a surjective homomorphism of the Doplicher-Roberts algebra $\mathcal{O}_{\rho}$ onto the corner $\mathrm{PC}^{*}\left(S_{x}\right) P$.

Proof: The algebra $\mathcal{O}_{\rho}$ is the $C^{*}$-enveloping algebra of the ${ }^{*}$-algebra ${ }^{0} \mathcal{O}_{\rho}$, so the homomorphism $\phi:{ }^{0} \mathcal{O}_{\rho} \rightarrow P B P \subset P C^{*}\left(S_{x}\right) P$ is by definition continuous, and extends to a homomorphism of $\mathcal{O}_{\rho}$ into $P C^{*}\left(S_{x}\right) P$. Since $\phi$ maps ${ }^{0} \mathcal{O}_{\rho}$ onto $P B P$, which is dense in $P C^{*}\left(S_{x}\right) P$, and homomorphisms between $C^{*}$-algebras have closed range, the Corollary follows. 
Corollary 2.3. Suppose $\rho$ is a representation of a compact group $G$ in $S U_{n}(\mathrm{C})$, for some $n>1$. If $\left\{S_{x}\right\}, P$ are as in Theorem 2.1, then $\mathcal{O}_{p}$ is isomorphic to $P C^{*}\left(S_{x}\right) P$.

Proof: By Theorem 2.12 of [3], there is a unique $C^{*}$-seminorm on ${ }^{0} \mathcal{O}_{\rho}$, which is actually a $C^{*}$-norm. Since pulling back the operator norm along the homomorphism of ${ }^{0} \mathcal{O}_{\rho}$ onto $P B P$ induces such a seminorm, we deduce that the homomorphism is isometric, and extends to an isomorphism of $\mathcal{O}_{\rho}$ onto the closure $P C^{*}\left(S_{x}\right) P$ of $P B P$. D

REMARK 2.4.. When the group is finite, the matrix $A_{\rho}$ is finite, and it follows from [2] that $C^{*}\left(S_{x}\right)=\mathcal{O}_{A_{\rho}}$ is simple (see Lemma 3.1 below). As the corner $\mathcal{O}_{\rho}$ is then necessarily full, we can deduce from [3, Corollary 2.3], and [2] that $K_{*}\left(\mathcal{O}_{\rho}\right) \cong K_{*}\left(\mathcal{O}_{A_{\rho}}\right)$ (we shall prove this again in Section 3 without appealing to [3] or requiring $\rho(G) \subset S U$ ). In principle, we can similarly deduce from [3] and [2] that $K_{*}\left(\mathcal{O}_{\rho}\right) \cong K_{*}\left(\mathcal{O}_{A_{\rho}}\right)$ when $G$ is compact and $\rho(G) \subset S U$, although some care will be needed in applying [2] because $A_{\rho}$ is infinite if $G$ is. However, since the calculation of $K_{*}\left(\mathcal{O}_{A}\right)$ in [1] does not obviously apply to infinite $A$, further work is needed before this result can be useful, and we defer it for now.

\section{DOPLICHER-ROBERTS ALGEBRAS OF FINITE GROUPS}

Our goal here is to prove that, when $G$ is finite, the complete Doplicher-Roberts algebra $\mathcal{O}_{\rho}$ is isomorphic to a corner in the corresponding Cuntz-Krieger algebra $\mathcal{O}_{A_{\rho}}$. Before we can state our theorem, we need to check that the $\{0,1\}$-matrix $A_{\rho}$ is one for which $\mathcal{O}_{A_{p}}$ can be uniquely defined, up to isomorphism, as the $C^{*}$-algebra generated by a family of non-zero partial isometries $\left\{S_{x}: x \in E\right\}$ satisfying

$$
S_{x}^{*} S_{x}=\sum_{y \in E} A_{\rho}(x, y) S_{y} S_{y}^{*}
$$

Cuntz and Krieger gave a sufficient condition (I) on the $\{0,1\}$-matrix $A_{\rho}[2$, p.254; Theorem 2.13], and showed that if in addition $A_{\rho}$ is irreducible, then $\mathcal{O}_{A_{\rho}}$ is simple [2, 2.14]. Both these properties of $A_{\rho}$ reduce to standard facts about the representation theory of finite groups:

LEMMA 3.1. If $\rho$ is a representation of a finite group and $1<\operatorname{dim} \rho<\infty$, then $A_{\rho}$ is irreducible and satisfies the Cuntz-Krieger condition (I).

Proof: We may as well suppose $\rho$ is faithful: if not, replace the group $G$ by $G / \operatorname{ker} \rho$. Then every irreducible representation of $G$ is contained in some tensor power of $\rho[5,(4.3)$ and (2.9)], and hence $R=\widehat{G}$; equivalently, for each $\pi \in \widehat{G}$ there is a path in the infinite diagram starting at $\iota$ and finishing at $\pi$. If $\pi_{c}$ is the contragredient representation $s \rightarrow\left(\pi_{s}-1\right)^{t}$, then $\iota$ is a summand of $\pi \otimes \pi_{c}$ (since the corresponding 
characters satisfy $\chi_{\pi_{c}}=\bar{\chi}_{\pi}$, this follows from [5, p.48 and (2.9)]), and hence for any $\pi \in \widehat{G}$ there is a path from $\pi$ to $\iota$. Putting these last two observations together gives a path joining $\iota$ to itself passing through any given $\pi$, and hence paths joining any given $\pi_{1}$ to any other $\pi_{2}$. Now given $x, y \in E$, we can use a path from $r(x)$ to $s(y)$ to produce a path starting with $x$ and finishing with $y$, and thus $A_{\rho}$ is irreducible. To see that $A_{\rho}$ satisfies (I) we just have to produce two different paths starting and finishing with the same edge $x$ : for then the irreducibility of $A_{\rho}$ implies that we can connect any other $y \in E$ to $x$. But if $\pi$ has maximal dimension, $\operatorname{dim} \rho \geqslant 2$ implies that $\pi \otimes \rho$ must have at least two irreducible summands, and hence that there are at least two edges $y, z$ with $\pi=s(y)=s(z)$. Now we take $x$ to be any edge with $r(x)=\pi$, and joining $r(y)$ and $r(z)$ to $s(x)$ gives two distinct paths starting and ending at $x$.

REMARK 3.2. The result always fails if $\operatorname{dim} \rho=1$. For then $\rho$ is an isomorphism of $G / \operatorname{ker} \rho$ onto a finite cyclic subgroup of $\mathrm{T}$, the map $\gamma \rightarrow \gamma \rho$ is an automorphism of $(G / \operatorname{ker} \rho)^{\wedge}$, and the matrix $A_{\rho}$ is a permutation matrix, which never satisfies condition (I). However, since $\rho(G)$ is cyclic, so is $G / \operatorname{ker} \rho, \rho$ must generate $(G / \operatorname{ker} \rho)^{-}$, and the permutation matrix is irreducible.

We now fix a family $\left\{S_{x}: x \in E\right\}$ of non-zero partial isometries on a Hilbert space $H$ satisfying (3.1), view $\mathcal{O}_{A_{\rho}}$ as $C^{*}\left(S_{x}: x \in E\right)$, and let

$$
P=\sum_{\{x \in E: s(x)=i\}} S_{x} S_{x}^{*}
$$

Our main result is:

THEOREM 3.3. Let $\rho$ be a representation of a finite group with $1<\operatorname{dim} \rho<\infty$. Then $\mathcal{O}_{\rho}$ is isomorphic to the corner $P \mathcal{O}_{A_{\rho}} P$.

We first have to establish the algebraic version. For it, we resume the notation of Sections 1 and 2.

Lemma 3.4. Suppose $G$ is finite and $1<\operatorname{dim} \rho<\infty$. Then the homomorphism $\phi$ of Theorem 2.1 is an isomorphism of ${ }^{0} \mathcal{O}_{\rho}$ onto PBP.

Proof: We begin by letting

$$
B_{m, n}=\operatorname{sp}\left\{S_{z} S_{y}^{*}:|x|=m,|y|=n, r\left(x_{m}\right)=r\left(y_{n}\right)\right\},
$$

so that by definition $\phi_{m, n}$ maps $\left(\rho^{m}, \rho^{n}\right)$ onto $P B_{m, n} P$ (recall that $P S_{x} S_{y}^{*} P=S_{x} S_{y}^{*}$ or 0 , so $P B_{m, n} P$ is spanned by those $S_{x} S_{y}^{*}$ where $\left.s\left(x_{1}\right)=s\left(y_{1}\right)=\iota\right)$. In fact we claim that the generators $S_{x} S_{y}^{*}$ for $B_{m, n}$ are linearly independent, so that $\phi_{m, n}$ is a linear isomorphism. To see why, suppose $\sum_{|x|=m,|y|=n} \lambda_{x, y} S_{x} S_{y}^{*}=0$ in $B$. If $|w|=m,|z|=n$ 
then $S_{w}^{*} S_{x}=\delta_{x, w} S_{x_{m}}^{*} S_{x_{m}}[2,2.1]$, and hence

$$
S_{w}^{*}\left(\sum_{|x|=m,|y|=n} \lambda_{x, y} S_{x} S_{y}^{*}\right) S_{z}=\lambda_{w, z} S_{w_{m}}^{*} S_{w_{m}} S_{z_{n}}^{*} S_{z_{n}}
$$

Thus $\lambda_{w, z}=0$ whenever $|w|=m,|z|=n$ and $\dot{r}\left(w_{m}\right)=r\left(z_{n}\right)$, and the $S_{x} S_{y}^{*}$ in $B_{m, n}$ are independent, as claimed.

The direct limit of the isomorphisms $\left\{\phi_{m, n}\right\}$ is an isomorphism

$$
\phi^{k}:{ }^{0} \mathcal{O}_{\rho}^{k}=\underline{\lim }\left(\rho^{p}, \rho^{p+k}\right) \rightarrow P B^{k} P=\underline{\lim } P B_{p, p+k} P=\bigcup_{p} P B_{p, p+k} P,
$$

and to show the direct sum $\phi=\oplus \phi^{k}$ is an isomorphism, it is enough to show that the range $P B P$ is the (algebraic) direct sum of the subspaces $P B^{k} P$. This is a highly nontrivial property of the algebra $\mathcal{O}_{A_{\rho}}=C^{*}\left(S_{x}\right)$, essentially established by Cuntz and Krieger in $[2,2.8,2.9]$, and is only true because the matrix $A_{\rho}$ satisfies condition (I) by Lemma 3.1 .

As shown in [2, bottorn of p.255], every $X$ in $B$ can be written in the form

$$
\sum_{k=-M}^{-1}\left(\sum_{|x|=|k|} S_{x} X_{x}\right)+X_{0}+\sum_{k=1}^{N}\left(\sum_{|y|=k} X_{y} S_{y}^{*}\right)
$$

where $X_{0}, X_{z}, X_{y}$ are all linear combinations of elements $S_{w} S_{z}^{*}$ with $|w|=|z|$. Since, for example, $\sum_{|y|=k} X_{y} S_{y}^{*} \in B^{k}$, and the recipe given in [2] shows that $X_{y} S_{y}^{*}$ lies in $P B^{k} P$ when $X \in P B P$, our problem is to show that this expression is unique. So suppose we have written 0 as a sum

$$
\sum_{k=-M}^{N} Z_{k}=\sum_{k=-M}^{-1}\left(\sum_{|x|=|k|} S_{x} Z_{x}\right)+Z_{0}+\sum_{k=1}^{N}\left(\sum_{|y|=k} Z_{y} S_{y}^{*}\right)
$$

If $Z$ denotes the formal sum on the right-hand side, then $Z=0$ implies $Z^{*} Z=0$, and hence, by $[2,2.8]$, that the homogeneous term $\left(Z^{*} Z\right)_{0} \in B^{0}$ vanishes. But this term is

$$
\begin{aligned}
\sum_{k=-M}^{-1}( & \left.\sum_{|x|=|k|=\left|x^{\prime}\right|} Z_{x}^{*} S_{x}^{*} S_{x^{\prime}} Z_{x^{\prime}}\right)+Z_{0}^{*} Z_{0}+\sum_{k=1}^{N}\left(\sum_{|y|=k} Z_{y} S_{y}^{*}\right)^{*}\left(\sum_{\left|y^{\prime}\right|=k} Z_{y^{\prime}} S_{y^{\prime}}^{*}\right) \\
= & \sum_{k=-M}^{-1}\left(\sum_{|x|=|k|} Z_{x}^{*} S_{x}^{*} S_{x} Z_{x}\right)+Z_{0}^{*} Z_{0}+\sum_{k=1}^{N}\left(\sum_{|y|=k} Z_{y} S_{y}^{*}\right)^{*}\left(\sum_{|y|=k} Z_{y} S_{y}^{*}\right) .
\end{aligned}
$$


Because the sum of positive operators can be 0 only if each term is 0 , we can deduce from this that $Z_{0}=0$ and $S_{x} Z_{x}=0$ for each $x$, and hence that $Z_{k}=0$ for $k<0$. The same argument using $Z Z^{*}=0$ gives $Z_{y} S_{y}^{*}=0$ for each $y$, so that $Z_{k}=0$ for $k>0$. We have shown that, algebraically at least, $B=\bigoplus_{k \in \mathbf{Z}} B^{k}$ and $P B P=\bigoplus_{k \in \mathbf{Z}} P B^{k} P$, and it follows that $\phi=\oplus \phi^{k}$ is an isomorphism, as required.

Proof of Theorem 3.3: Cuntz and Krieger prove the uniqueness of $\mathcal{O}_{A}$ by showing that the *-algebra $B$ generated by the partial isometries has a unique $C^{*}$ norm $\|\cdot\|_{B}$, namely that coming from its action on $H$. Since we know from the Lemma that ${ }^{0} \mathcal{O}_{\rho}$ is ${ }^{*}$-isomorphic to $P B P$, our problem is to show that the enveloping $C^{*}$. norm $\|\cdot\|_{C^{*}}$ on $P B P$ coincides with $\|\cdot\|_{B}$ on $P B P$. We certainly have $\|\cdot\|_{B} \leqslant\|\cdot\|_{C^{*}}$, so it will be enough to show that, for any *-representation $\pi$ of $P B P$, there is a *-representation $\tau$ of $B$ such that $\|\pi(Y)\| \leqslant\|\tau(Y)\|$ for $Y \in P B P$; if so, then

forces

$$
\begin{aligned}
\|Y\|_{B} & =\|\operatorname{id} \oplus \tau(Y)\|=\sup \left\{\|Y\|_{B},\|\tau(Y)\|\right\} \\
\|Y\|_{C^{*}} & =\sup \left\{\|\pi(Y)\|: \pi \text { is a }{ }^{*} \text {-representation of } P B P\right\} \\
& \leqslant \sup \left\{\|\tau(Y)\|: \tau \text { is a }{ }^{*} \text {-representation of } B\right\} \\
& \leqslant\|Y\|_{B} .
\end{aligned}
$$

Given $\pi$, we intend to write down a formula for such a $\tau$, but we need to do some background work first.

For each edge $x$, we choose a path $\alpha(x)$ starting at the vertex $\iota$ and ending at $x$ : if $s(x)=\iota$, we insist that $\alpha(x)$ consists of the single edge $x$. We then define $R_{x}=S_{x} S_{\alpha(x)}^{*}$, so that if $s(x)=\iota$, we have $R_{x}=S_{x} S_{x}^{*}$, and in general, $R_{x}$ is a partial isometry with initial projection $R_{x}^{*} R_{x} \leqslant P$. For single edges $w, z$ we have $S_{w}^{*} S_{z}=0$ unless $w=z$, and therefore

$$
\begin{aligned}
S_{z}^{*} S_{y}^{*} S_{y} S_{z} & =S_{z}^{*}\left(\sum_{w} A(y, w) S_{w} S_{w}^{*}\right) S_{z} \\
& =A(y, z) S_{z} S_{z}^{*}
\end{aligned}
$$

which is 0 or $S_{z} S_{z}^{*}$; since we know $\alpha(x)$ is a path, $S_{\alpha(x)} \neq 0$ and cancellation from the centre out shows

$$
\begin{aligned}
R_{x} R_{x}^{*} & =S_{x}\left(S_{x}^{*} \cdots S_{\alpha(x)_{j}}^{*} \cdots S_{\alpha(x)_{1}}^{*}\right)\left(S_{\alpha(x)_{1}} \cdots S_{\alpha(x)} \cdots S_{x}\right) S_{x}^{*} \\
& =S_{x}\left(S_{x}^{*} S_{x}\right) S_{x}^{*} \\
& =S_{x} S_{x}^{*}
\end{aligned}
$$

Thus we have

$$
1=\sum_{x \in E} S_{x} S_{x}^{*}=\sum_{x \in E} R_{x} R_{x}^{*}
$$


We now define $\tau: B \rightarrow B\left(H^{E}\right)=M_{E}(B(H))$ by letting $\tau(Y)$ be the $E \times E$ matrix with $(x, y)$-entry $\tau(Y)_{x, y}=\pi\left(R_{x}^{*} Y R_{y}\right)$; because both $R_{x}^{*} R_{x}$ and $R_{y}^{*} R_{y}$ are dominated by $P, R_{x}^{*} Y R_{y}$ lies in $P B P$, and we can legitimately apply $\pi$ to it. We claim $\tau$ is a *-homomorphism: it is clearly linear, equation (3.1) implies that it is multiplicative:

$$
\begin{aligned}
(\tau(Y) \tau(Z))_{x, z} & =\sum_{y} \pi\left(R_{x}^{*} Y R_{y}\right) \pi\left(R_{y}^{*} Z R_{z}\right) \\
& =\pi\left(R_{x}^{*} Y\left(\sum_{y} R_{y} R_{y}^{*}\right) Z R_{z}\right) \\
& =\pi\left(R_{x}^{*}(Y Z) R_{z}\right) \\
& =\tau(Y Z)_{x, z},
\end{aligned}
$$

and it is easily seen to preserve adjoints:

$$
\left(\tau(Y)^{*}\right)_{x, z}=\left(\tau(Y)_{z, x}\right)^{*}=\pi\left(R_{z}^{*} Y R_{x}\right)^{*}=\pi\left(R_{z}^{*} Y^{*} R_{z}\right)=\tau\left(Y^{*}\right)_{x, z} .
$$

Finally, note that because $R_{x}=S_{x} S_{x}^{*}$ when $x \in I=\{x \in E: s(x)=\iota\}$, we have $P=$ $\sum_{x \in I} R_{x}=\sum_{x \in I} R_{x}^{*}$, and hence for $Y \in P B P$

$$
\pi(Y)=\sum_{x, y \in I} \pi\left(R_{x}^{*} Y R_{y}\right)
$$

Since the ranges of the partial isometries $R_{y}$ are mutually orthogonal, the norm of this sum is equal to the norm of the $I \times I$ matrix

$$
\left(\pi\left(R_{x}^{*} Y R_{y}\right)\right)_{x, y \in I} \in M_{I}(B(H)) ;
$$

but this is a submatrix of the $E \times E$ matrix $\tau(Y)$, and hence

$$
\|\pi(Y)\|=\left\|\left(\pi\left(R_{x}^{*} Y R_{y}\right)\right)_{x, y \in I}\right\| \leqslant\|\tau(Y)\|
$$

as required.

COROLLARY 3.5. For any representation $\rho$ of a finite group satisfying $1<$ $\operatorname{dim} \rho<\infty, \mathcal{O}_{\rho}$ is a simple $C^{*}$-algebra which is Morita equivalent to the corresponding $\mathcal{O}_{A_{\rho}}$.

Proof: We have already shown that $A=A_{\rho}$ is irreducible and satisfies condition (I), so $\mathcal{O}_{A}$ is simple by [2, Theorem 2.14]. Thus the corner $P \mathcal{O}_{A} P$ is full - there is no nontrivial ideal which can contain it. This implies that the $\mathcal{O}_{A}-P \mathcal{O}_{A} P$ bimodule $\mathcal{O}_{A} P$ is an imprimitivity bimodule with the inner products

$$
\begin{gathered}
\langle X P, Y P\rangle_{P \mathcal{O}_{A} P}=P X^{*} Y P, \\
\mathcal{O}_{A}\langle X P, Y P\rangle=X P Y^{*}
\end{gathered}
$$

the fullness of $P \mathcal{O}_{A} P$ says precisely that the span of the range of the $\mathcal{O}_{A}$-valued inner product is dense in $\mathcal{O}_{A}$. Thus the result follows from the Theorem. 


\section{The $K$-Theory of Doplicher-Roberts algebras}

We want to compute the $K$-theory of a Doplicher-Roberts algebra $\mathcal{O}_{\rho}$ using Cuntz's computation of $K_{*}\left(\mathcal{O}_{A_{\rho}}\right)$, which is isomorphic to $K_{*}\left(\mathcal{O}_{\rho}\right)$ because the $C^{*}$-algebras are Morita equivalent. The key result is $\left[1\right.$, Proposition 3.1], which asserts that $K_{0}\left(\mathcal{O}_{A}\right)$ and $K_{1}\left(\mathcal{O}_{A}\right)$ are, respectively, the cokernel and kernel of the map $1-A^{t}: \mathbf{Z}^{E} \rightarrow \mathbf{Z}^{E}$. Now when we constructed $A_{\rho}$ from the bipartite graph, we chose to use the set $E$ of edges rather than the set $R$ of vertices as our index set. This has the advantage that $A_{\rho}$ is always a $\{0,1\}$-matrix, as opposed to an integer matrix, but the disadvantage that $E$ is usually a lot bigger than $R$, which makes calculations messier. So we want to first show that either matrix can be used in our calculation of $K$-theory. In fact this is quite generally true: if $A, B$ are the two matrices associated to any bipartite graph, then $1-A^{t}, 1-B^{t}$ have the same kernel and cokernel, and if both are $\{0,1\}$-matrices, they give isomorphic Cuntz-Krieger algebras. These facts are surely well-known - for example, they are implicit in the way Cuntz and Krieger handle general integer matrices $[2,2.16]$ - but we do not know where the details have been written down.

Suppose, then, that we have a bipartite graph with vertices $V$, edges $E$ and range, source maps $r, s: E \rightarrow R$. We define

$$
\begin{aligned}
& B(i, j)=\#\{x \in E: s(x)=i, r(x)=j\} \\
& A(x, y)= \begin{cases}1 & \text { if } r(x)=s(y) \\
0 & \text { otherwise. }\end{cases}
\end{aligned}
$$

Proposition 4.1. (1) If $B$ is a $\{0,1\}$-matrix satisfying (I), then $A$ satisfies (I) and $\mathcal{O}_{B} \cong \mathcal{O}_{A}$.

(2) There are isomorphisms

$$
\begin{aligned}
\operatorname{ker}\left(\left(1-B^{t}\right): \mathbf{Z}^{V} \rightarrow \mathbf{Z}^{V}\right) & \cong \operatorname{ker}\left(\left(1-A^{t}\right): \mathbf{Z}^{E} \rightarrow \mathbf{Z}^{E}\right) \\
\mathbf{Z}^{V} /\left(1-B^{t}\right)\left(\mathbf{Z}^{V}\right) & \cong \mathbf{Z}^{E} /\left(1-A^{t}\right)\left(\mathbf{Z}^{E}\right)
\end{aligned}
$$

Proof: If $B$ has entries in $\{0,1\}$, paths of vertices are essentially the same as paths of edges, and the first assertion is essentially clear. For the second, suppose $S_{i}$ are partial isometries satisfying

$$
S_{i}^{*} S_{i}=\sum_{j \in V} B(i, j) S_{j} S_{j}^{*}
$$

and define $T_{x}=S_{s(x)} S_{r(x)} S_{r(x)}^{*}$. Then certainly each $T_{x}$ is a partial isometry in $C^{*}\left(S_{i}\right)$ 
and

$$
\begin{aligned}
S_{i}=S_{i} S_{i}^{*} S_{i} & =\sum_{j \in V} B(i, j) S_{i} S_{j} S_{j}^{*} \\
& =\sum_{\{j: B(i, j)=1\}} S_{i} S_{j} S_{j}^{*} \\
& =\sum_{\{x: s(x)=i\}} S_{s(x)} S_{r(x)} S_{r(x)}^{*},
\end{aligned}
$$

since $B(i, j)=1$ if and only if there is is an edge $x$ from $i$ to $j$. Thus $C^{*}\left(S_{i}\right)=C^{*}\left(T_{x}\right)$. We now verify that the $T_{x}$ generate $\mathcal{O}_{A}$. On the one hand,

$$
\begin{aligned}
\sum_{y \in E} A(x, y) T_{y} T_{y}^{*} & =\sum_{\{y: s(y)=r(x)\}} S_{\lrcorner(y)}\left(S_{r(y)} S_{r(y)}^{*}\right)^{2} S_{s(y)}^{*} \\
& =S_{r(x)}\left(\sum_{\{y: s(y)=r(x)\}} S_{r(y)} S_{r(y)}^{*}\right) S_{r(x)}^{*} \\
& =S_{r(x)}\left(\sum_{\{j: B(r(x), j)=1\}} S_{j} S_{j}^{*}\right) S_{r(x)}^{*} \\
& =S_{r(x)}\left(S_{r(x)}^{*} S_{r(x)}\right) S_{r(x)}^{*} \\
& =S_{r(x)} S_{r(x)}^{*} ;
\end{aligned}
$$

on the other, since the $S_{i}$ have mutually orthogonal ranges, we also have

$$
\begin{aligned}
T_{x}^{*} T_{x} & =S_{r(x)} S_{r(x)}^{*}\left(S_{s(x)}^{*} S_{\triangleleft(x)}\right) S_{r(x)} S_{r(x)}^{*} \\
& =S_{r(x)} S_{r(x)}^{*}\left(\sum_{\{j: B(s(x), j)=1\}} S_{j} S_{j}^{*}\right) S_{r(x)} S_{r(x)}^{*} \\
& =S_{r(x)} S_{r(x)}^{*},
\end{aligned}
$$

so the $T_{x}$ do satisfy the Cuntz-Krieger relations for $A$. Thus by the Cuntz-Krieger uniqueness theorem we have

$$
\mathcal{O}_{B} \cong C^{*}\left(S_{i}\right)=C^{*}\left(T_{x}\right) \cong \mathcal{O}_{A},
$$

giving (1).

To establish (2), we use the source and range maps to define $V \times E$ and $E \times V$ matrices:

$$
\begin{aligned}
& S(i, x)= \begin{cases}1 & \text { if } s(x)=i \\
0 & \text { otherwise }\end{cases} \\
& R(x, i)= \begin{cases}1 & \text { if } r(x)=i \\
0 & \text { otherwise }\end{cases}
\end{aligned}
$$


We have

$$
(R S)(x, y)=\sum_{i} R(x, i) S(i, y)
$$

and since each summand is 0 or 1 ,

$$
\begin{aligned}
R(x, i) S(i, y)=1 & \Leftrightarrow R(x, i)=1=S(i, y) \\
& \Leftrightarrow r(x)=i=s(y) .
\end{aligned}
$$

For each fixed pair $(x, y)$, this can happen for exactly one $i$, and hence we can deduce that $R S=A$. Similarly,

$$
\begin{aligned}
(S R)(i, j) & =\sum_{x} S(i, x) R(x, j) \\
& =\#\{x \in E: S(i, x)=1=R(x, j)\} \\
& =\#\{x \in E: s(x)=i, r(x)=j\}
\end{aligned}
$$

and $S R=B$. Of course, we also have $R^{t} S^{t}=B^{t}, S^{t} R^{t}=A^{t}$, and hence the following standard lemma gives what we need:

LEMma 4.2. Suppose $R, S$ are $V \times E, E \times V$ matrices with entries in $\{0,1\}$, and $B=R S \in M_{V}(\mathbf{Z}), A=S R \in M_{E}(\mathbf{Z})$. Then the transformation $S: \mathbf{Z}^{V} \rightarrow \mathbf{Z}^{E}$ induces isomorphisms of $\operatorname{ker}\left((1-B): \mathbf{Z}^{V} \rightarrow \mathbf{Z}^{V}\right)$ onto $\operatorname{ker}(1-A)$, and coker $(1-B)$ $=\mathbf{Z}^{V} /(1-B)\left(\mathbf{Z}^{V}\right)$ onto coker $(1-A)$.

Proof: We first observe that, for each $\lambda \neq 0, S: \mathbf{R}^{V} \rightarrow \mathbf{R}^{E}$ is an isomorphism of the eigenspace

$$
E_{\lambda}^{B}=\left\{v \in \mathbf{R}^{V}: B v=\lambda v\right\}
$$

onto $E_{\lambda}^{A} \subset \mathbf{R}^{E}$, with inverse given by $\lambda^{-1} R$. Since both $R, S$ have integer entries, it follows that $S$ restricts to an isomorphism of $\operatorname{ker}(1-B)=E_{1}^{B} \cap \mathrm{Z}^{V}$ onto $\operatorname{ker}(1-A)=$ $E_{1}^{A} \cap \mathbf{Z}^{E}$ with inverse $R$. Next, we note that if $z \in \operatorname{im}(1-B)$, say $z=(1-B) v$, then

$$
S z=S(1-R S) v=(1-S R) S v=(1-A)(S v),
$$

so $S$ does map $\operatorname{im}(1-B)$ into $\operatorname{im}(1-A)$, and induces a homomorphism $\phi$ of $\operatorname{coker}(1-B)$ into coker $(1-A)$. In the same way, $R$ induces a homomorphism $\psi$ of coker $(1-A)$ into coker $(1-B)$, which we claim is an inverse for $\phi$. For

$$
\begin{aligned}
\psi \circ \phi(v+\operatorname{im}(1-B)) & =S R v+\operatorname{im}(1-B) \\
& =v-(v-S R v)+\operatorname{im}(1-B) \\
& =v+\operatorname{im}(1-B),
\end{aligned}
$$

and similarly $\phi \circ \psi$ is the identity on $\operatorname{coker}(1-A)$.

This lemma completes the proof of Proposition 4.1. 
EXAmpLe 4.3. $G=S_{3}$. The character table of $S_{3}$ is

e

\begin{tabular}{lrrr}
\hline$:$ & 1 & 1 & 1 \\
$\sigma:$ & 1 & -1 & 1 \\
$\pi:$ & 2 & 0 & -1
\end{tabular}

The obvious representation to take for $\rho$ is the 2-dimensional representation $\pi$ : it is faithful because

$$
\operatorname{ker} \pi=\left\{s \in G: \chi_{\pi}(s)=\chi_{\pi}(e)=2\right\}=\{e\}
$$

$[5,(2.19)]$. We trivially have $\iota^{2}=\iota, \iota \otimes \sigma=\sigma, \iota \otimes \pi \sim \pi$, and $\sigma^{2}=\iota$; the characters of the other tensor products are given by

$$
\begin{gathered}
\chi_{\sigma \otimes \pi}=\chi_{\sigma} \chi_{\pi}=\chi_{\pi}, \quad \text { and } \\
\chi_{\pi \otimes \pi}=\left(\chi_{\pi}\right)^{2}=\chi_{\iota}+\chi_{\sigma}+\chi_{\pi},
\end{gathered}
$$

and since the decomposition of the character determines the decomposition of the representation $[5,(2.9)]$, we have $\sigma \otimes \pi \sim \pi$ and $\pi^{2} \sim \iota \oplus \pi \oplus \sigma$. We therefore have

$$
B_{\pi}=\left(\begin{array}{lll}
0 & 1 & 0 \\
1 & 1 & 1 \\
0 & 1 & 0
\end{array}\right) \quad \text { and } \quad 1-B_{\pi}^{\boldsymbol{t}}=\left(\begin{array}{rrr}
1 & -1 & 0 \\
-1 & 0 & -1 \\
0 & -1 & 1
\end{array}\right) .
$$

Since $\operatorname{det}\left(1-B_{\pi}^{t}\right)=2, \operatorname{ker}\left(1-B_{\pi}^{t}\right)=0$ and $K_{1}\left(\mathcal{O}_{\pi}\right) \cong K_{1}\left(\mathcal{O}_{B_{\pi}}\right)=0$. However, for $(m, n, p) \in \mathbf{Z}^{3}$, the unique solution $v$ of $\left(1-B^{t}\right) v=(m, n, p)$ in $\mathbf{R}^{3}$ is

$$
v=\left(\frac{m-n-p}{2}, \frac{-m-n-p}{2}, \frac{-m-n+p}{2}\right),
$$

which lies in $\mathbf{Z}^{3}$ if and only if $m+n+p \in 2 \mathbf{Z}$. Thus

$$
(m, n, p) \rightarrow(m+n+p)+2 Z
$$

induces an isomorphism of $K_{0}\left(\mathcal{O}_{\pi}\right) \cong K_{0}\left(\mathcal{O}_{B_{\pi}}\right) \cong \mathbf{Z}^{3} /\left(1-B_{\pi}^{t}\right)\left(\mathbf{Z}^{3}\right)$ onto $\mathbf{Z}_{2}$.

If we take for $\rho$ the faithful representation $\pi \oplus \iota$, we have instead

$$
B_{\rho}=\left(\begin{array}{lll}
1 & 1 & 0 \\
1 & 2 & 1 \\
0 & 1 & 1
\end{array}\right) \quad \text { and } \quad 1-B_{\rho}^{t}=\left(\begin{array}{rrr}
0 & -1 & 0 \\
-1 & -1 & -1 \\
0 & -1 & 0
\end{array}\right)
$$


Thus for this choice of $\rho$,

$$
K_{1}\left(\mathcal{O}_{\rho}\right) \cong K_{1}\left(\mathcal{O}_{B_{\rho}}\right) \cong \operatorname{ker}\left(1-B_{\rho}^{t}\right) \cong \mathbf{Z},
$$

and the map $(m, n, p) \rightarrow m-p$ induces an isomorphism

$$
K_{0}\left(\mathcal{O}_{\rho}\right) \cong K_{0}\left(\mathcal{O}_{B_{\rho}}\right) \cong \mathbf{Z}^{3} /\left(1-B_{\rho}^{t}\right)\left(\mathbf{Z}^{3}\right) \cong \mathbf{Z}
$$

Alternatively, if $\rho=\pi \oplus \sigma$, we have

$$
1-B_{\rho}^{t}=\left(\begin{array}{rrr}
1 & -1 & -1 \\
-1 & -1 & -1 \\
-1 & -1 & 1
\end{array}\right)
$$

Here $\operatorname{det}\left(1-B_{\rho}^{t}\right)=-4$, so $K_{1}\left(\mathcal{O}_{\rho}\right)=0$, but $\left(1-B_{\rho}^{t}\right) v=(m, n, p)$ has solution

$$
v=\left(\frac{m-n}{2}, \frac{-m-p}{2}, p-n\right)
$$

and $(m, n, p) \rightarrow(m-n,-m-p)$ induces an isomorphism of coker $\left(1-B_{\rho}^{t}\right) \cong K_{0}\left(\mathcal{O}_{\rho}\right)$ onto $\mathbf{Z}_{2} \times \mathbf{Z}_{2}$.

EXAMPLE 4.4. $G=A_{5} \cong P S L(2,5) \cong S L(2,4)$. It is important in the work of Doplicher and Roberts that the representation $\rho$ is faithful and special unitary, and we shall now discuss an example where there are several irreducible representations of this kind - indeed, since this group has only the trivial one-dimensional representation, $s \rightarrow \operatorname{det} \pi(s)$ is always identically 1 , and any representation is special unitary. We write $\pi_{i}(1 \leqslant i \leqslant 5)$ for the irreducible representations, with $\pi_{1}=\iota$, and $\chi_{i}$ for the corresponding characters. Then the character table for $A_{5}$ is:

\begin{tabular}{ccrrrr} 
& 1 & 2 & 3 & $5_{1}$ & $5_{2}$ \\
\hline$\chi_{1}=\iota:$ & 1 & 1 & 1 & 1 & 1 \\
$\chi_{2}:$ & 4 & 0 & 1 & -1 & -1 \\
$\chi_{3}:$ & 5 & 1 & -1 & 0 & 0 \\
$\chi_{4}:$ & 3 & -1 & 0 & $\alpha_{1}$ & $\alpha_{2}$ \\
$\chi_{5}:$ & 3 & -1 & 0 & $\alpha_{2}$ & $\alpha_{1}$
\end{tabular}

where $\alpha_{1}=(1+\sqrt{5}) / 2, \alpha_{2}=(1-\sqrt{5}) / 2$. Calculating as in the previous example with $\rho=\pi_{2}$ gives

$$
B_{2}=\left(\begin{array}{lllll}
0 & 1 & 0 & 0 & 0 \\
1 & 1 & 1 & 1 & 1 \\
0 & 1 & 2 & 1 & 1 \\
0 & 1 & 1 & 0 & 1 \\
0 & 1 & 1 & 1 & 0
\end{array}\right) \quad \text { and } \quad 1-B_{2}^{t}=\left(\begin{array}{rrrrr}
1 & -1 & 0 & 0 & 0 \\
-1 & 0 & -1 & -1 & -1 \\
0 & -1 & -1 & -1 & -1 \\
0 & -1 & -1 & 1 & -1 \\
0 & -1 & -1 & -1 & 1
\end{array}\right)
$$


The rank of $1-B_{2}^{t}$ is 4 , with

$$
K_{1}\left(\mathcal{O}_{\pi_{2}}\right) \cong \operatorname{ker}\left(1-B_{2}^{t}\right)=\{(n, n,-n, 0,0)\} \cong \mathbf{Z}
$$

Given $\mathbf{m}=(m, n, p, q, r) \in \mathbf{Z}^{5}$, the equation $\left(1-B_{2}^{t}\right) v=\mathbf{m}$ has a solution in $\mathbf{R}^{5}$ only if $p=n+m$, and then the solution space in $\mathbf{R}^{\mathbf{5}}$ is

$$
\left\{t(1,1,-1,0,0)+\left(m, 0, \frac{-q-r}{2}, \frac{q-p}{2}, \frac{r-p}{2}\right)\right\}
$$

it follows that

$$
(m, n, p, q, r) \rightarrow(m+n-p, q-p \bmod 2, r-p \bmod 2)
$$

induces an isomorphism of $K_{0}\left(\mathcal{O}_{\pi_{2}}\right) \cong \mathbf{Z}^{5} /\left(1-B_{2}^{t}\right)\left(\mathbf{Z}^{5}\right)$ onto $\mathbf{Z} \times \mathbf{Z}_{2} \times \mathbf{Z}_{2}$.

Next we take $\rho=\pi_{4}$. This time

$$
B_{4}=\left(\begin{array}{rrrrr}
0 & 0 & 0 & 1 & 0 \\
0 & 1 & 1 & 0 & 1 \\
0 & 1 & 1 & 1 & 1 \\
1 & 0 & 1 & 1 & 0 \\
0 & 1 & 1 & 0 & 0
\end{array}\right) \quad \text { and } \quad 1-B_{4}^{t}=\left(\begin{array}{rrrrr}
1 & 0 & 0 & -1 & 0 \\
0 & 0 & -1 & 0 & -1 \\
0 & -1 & 0 & -1 & -1 \\
-1 & 0 & -1 & 0 & 0 \\
0 & -1 & -1 & 0 & 1
\end{array}\right)
$$

We have $\operatorname{det}\left(1-B_{4}^{t}\right)=4$, so $\operatorname{ker}\left(1-B_{4}^{t}\right)=0=K_{1}\left(\mathcal{O}_{\pi_{4}}\right)$, and if $\mathbf{m}=(m, n, p, q, r)$, then $\left(1-B_{4}^{t}\right) v=\mathbf{m}$ has unique solution

$$
\begin{aligned}
&\left(\frac{r-p-3 q+m+2 n}{4}, \frac{-r-p+q+m}{2}, \frac{-r+p-q-m-2 n}{4}\right. \\
&\left.\frac{r-p-3 q-3 m+2 n}{4}, \frac{r-p+q+m-2 n}{4}\right)
\end{aligned}
$$

which lies in $\mathbf{Z}^{5}$ if and only if $r-p-3 q+m+2 n \in 4 Z$; thus

$$
K_{0}\left(\mathcal{O}_{\pi_{4}}\right) \cong K_{0}\left(\mathcal{O}_{B_{4}}\right) \cong \mathbf{Z}^{5} /\left(1-B_{4}^{t}\right)\left(\mathbf{Z}^{5}\right) \cong \mathbf{Z} / 4 \mathbf{Z}
$$

In particular, the $K$-groups of $\mathcal{O}_{\pi_{4}}$ and $\mathcal{O}_{\pi_{2}}$ are quite different, even though both $\pi_{4}$ and $\pi_{2}$ are faithful, irreducible, special unitary representations of $A_{5}$.

\section{References}

[1] J. Cuntz, 'A class of $C^{*}$-algebras and topological Markov chains II: reducible chains and the Ext-functor for $C^{*}$-algebras', Invent. Math. 63 (1981), 25-40. 
[2] J. Cuntz and W. Krieger, 'A class of $C^{*}$-algebras and topological Markov chains', Invent. Math. 56 (1980), 251-268.

[3] S. Doplicher and J.E. Roberts, 'Duals of compact Lie groups realised in the Cuntz algebras and their actions on $C^{*}$-algebras', J. Funct. Anal. 74 (1987), 96-120.

[4] S. Doplicher and J.E. Roberts, 'Endomorphisms of $C^{*}$-algebras, cross products and duality for compact groups', Ann. of Math. 130 (1989), 75-119.

[5] I.M. Isaacs, Character theory of finite groups (Academic Press, New York, 1976).

[6] M.H. Mann, I. Raeburn and C.E. Sutherland, Representations of compact groups, Cuntz-Krieger algebras, and groupoid $C^{*}$-algebras, Proc. Centre Math. Appl. Austral. Nat. Univ. (to appear).

[7] G.Y.-L. Shiu and C.E. Sutherland, 'Groupoid models for AF-algebras', (submitted).

\section{M.H. Mann}

School of Mathematics

University of New South Wales

PO Box 1

Kensington NSW 2033

Australia

C.E. Sutherland

School of Mathematics

University of New South Wales

PO Box 1

Kensington NSW 2033

Australia

\section{Raeburn}

Department of Mathematics

University of Newcastle

Newcastle NSW 2308

Australia 